\title{
The spatial coherence wavelets and second-order correlation
}

\author{
Juan Manuel González-Toro
}

Departamento de Ciencias Naturales y Exactas, Fundación Universidad Autónoma de Colombia, Bogotá, Colombia

\begin{abstract}
A description of the second-order spatial coherence based on the theory of spatial coherence wavelets is presented. Such description is performed in the classical context of optical fields and chaotic sources. The concepts of radiant and virtual point sources are introduced. This theory suggests that the second-order spatial coherence state of light can be described in terms of three layers of point sources; a strategy that can increase the performance of numerical algorithms. The obtained modulation in coherence is similar to that measured in Hanbury-Brown and Twiss effect for binary stars.
\end{abstract}

Key words: Second-order spatial coherence, Hanbury-Brown and Twiss effect, Binary stars.

Onditas de coherencia espacial y correlación de segundo orden

\section{Resumen}

Se presenta una descripción de la coherencia espacial de segundo orden, basada en la teoría de onditas de coherencia espacial. Tal descripción es realizada en el contexto de campos ópticos y fuentes caóticas. Se introducen los conceptos de fuentes puntuales radiantes y virtuales. Esta teoría sugiere que el estado de coherencia espacial de segundo orden puede ser descrito en términos de tres capas de fuentes puntuales; una estrategia que puede mejorar el rendimiento de los algoritmos numéricos. La modulación obtenida en la coherencia es similar a la medida en el efecto Hanbury-Brown y Twiss para estrellas binarias.

Palabras clave: coherencia espacial de segundo orden, Efecto Hanbury-Brown y Twiss, estrellas binarias.

\section{Introduction}

The theory of spatial coherence wavelets provides a phasespace representation for classical optical phenomena within the scope of the first-order coherence (Castañeda, 2010a). In this theory, an optical field in any state of spatial coherence can be described by the emission of two types of point sources distributed in two different layers of space (Castañeda, 2010b).

The first type is called radiant point sources and they are located at first layer. These are responsible for the propagation of the radiant energy of the field, a positive definite quantity independent from the spatial coherence state and recordable by the squared-modulus detectors. The second type of sources is named virtual point sources and they are placed at the second layer. Their energies can take positive and negative values and they are not directly recordable by detectors, but they are crucial to describe diffraction and interference because they modulate the radiant energy by increasing and decreasing the local values without altering the value of the total energy of the field. Such energies depend on the spatial coherence state. A virtual point source is turned on at the midpoint of any pair of radiant point sources, not necessarily consecutive, within the structured spatial coherence support centered at that position
(Castañeda, 2010b), which implies that the set of radiant sources must be discrete. These virtual sources are called first-order virtual point sources. This model can completely describe the first-order spatial coherence properties of scalar wave fields.

Now, Young-like experiments with first-order virtual point sources are analyzed. The interference between contributions from these sources is a result of the secondorder state of spatial coherence of the field. It leads to correlation between the spatial coherence wavelets, which also involves the correlation of the cross-spectral densities. This development is performed in the classical context of optical fields and chaotic sources, which is appropriate for describing stars. The result is compared with that obtained in the Hanbury-Brown and Twiss effect to measure the angular separation of binary stars systems.

The study of this effect in the spatial domain is valuable, for instance, to construct modern intensity interferometers with Cherenkov telescopes (Le Bohec and Holder, 2006).

\footnotetext{
Corresponding author:

Juan Manuel González-Toro, jgonzalez.toro@fuac.edu.co

Recibido: 18 de Julio de 2015

Aceptado: 23 de septiembre de 2015
} 


\section{Spatial coherence wavelet}

Spatial coherence wavelet is defined as the basic vehicle for simultaneous transport of information about the energy (power spectrum) and the state of the first-order spatial coherence of optical field (correlation between the complex amplitudes of the field at two different points of the space) from the aperture plane $(A P)$ to the observation plane $(O P)$ (Castañeda, 2010a). The planes are separated by a distance $z$. Center-difference coordinates in the $A P\left(\xi_{A}, \xi_{D}\right)$ and the $O P\left(r_{A}, r_{D}\right)$ are used in order to denote pairs of points at the positions $\left(\xi_{A}+\xi_{D} / 2, \xi_{A}-\xi_{D} / 2\right)$ and $\left(r_{A}+r_{D} / 2, r_{A}-r_{D} / 2\right)$, as illustrated in Figure 1. These points are simplified as $\left(\xi_{A} \pm\right.$ $\left.\xi_{D} / 2\right)$ and $\left(r_{A} \pm r_{D} / 2\right)$, respectively.

The radiant and virtual point sources are located at the $A P$, each of these in the corresponding layer. $\xi_{D}$ is the separation vector between radiant point sources. The wavelet is denoted as:

$$
\mathbf{W}\left(\mathbf{r}_{A} \pm \frac{\mathbf{r}_{D}}{2} ; \xi_{A}\right)=\mathbf{S}\left(\xi_{A}, \mathbf{r}_{A}\right) \exp \left[-i \frac{k}{Z}\left(\xi_{A} \cdot \mathbf{r}_{D}\right)\right],
$$

with wave-number $k=2 \pi / \lambda$, wavelength $\lambda$ and $\mathbf{S}\left(\xi_{A}, r_{A}\right)$ the marginal power spectrum. It is a Wigner distribution function with energy units, defined as (Castañeda, 2010a):

$$
\begin{aligned}
\mathbf{S}\left(\xi_{A}, \mathbf{r}_{A}\right)= & \int_{A P} W\left(\xi_{A} \pm \frac{\xi_{D}}{2}\right) \\
& \exp \left(i \frac{k}{Z} \xi_{A} \cdot \xi_{D}\right) \exp \left(-i \frac{k}{Z} \xi_{D} \cdot \mathbf{r}_{A}\right) d^{2} \xi,
\end{aligned}
$$

where $W\left(\xi_{A} \pm \xi_{D} / 2\right)$ is the cross-spectral density of the field at the $A P$, referred to the structured spatial coherence support centered at $\xi_{A}$ that encloses the pairs of radiant point sources with separation vectors $\xi_{D}$. The cross-spectral density $W$ provides a measure of statistical similarity between light fluctuations at two points of space-time, which is a measure of the correlation between the complex

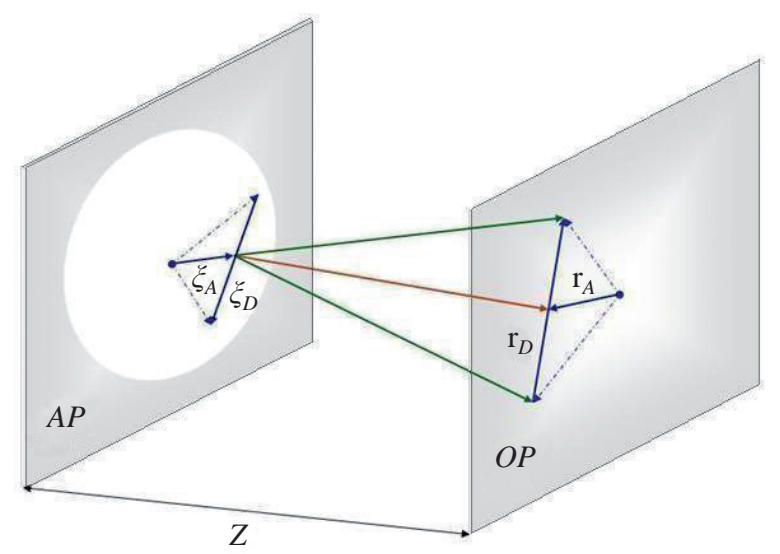

Figure 1. Illustration of the center-difference coordinates $\left(\xi_{A}, \xi_{D}\right)$ and $\left(\mathrm{r}_{A}, \mathrm{r}_{D}\right)$ at the aperture plane $(A P)$ and the observation plane $(O P)$, respectively. amplitudes on the component frequency spectrum $\omega$ of light vibrations at these points (Mandel and Wolf, 1995). These amplitudes are described by stationary random processes. Such processes have the characteristic that their statistical moments are independent of time, although their values are random variables that fluctuate over time (Goodman, 2000). Since the correlation between the amplitudes is an ensemble average, then its value does not depend on time but on the points in space where the correlation is evaluated, i.e. the correlation is a pure spatial descriptor.

The superposition of spatial coherence wavelets produces the cross-spectral density of the field at the $O P$ :

$$
W\left(\mathbf{r}_{A} \pm \frac{\mathbf{r}_{D}}{2}\right)=\left(\frac{1}{\lambda z}\right)^{2} \exp \left[i \frac{k}{z} \mathbf{r}_{A} \cdot \mathbf{r}_{D}\right] \int_{A P} \mathbf{W}\left(\mathbf{r}_{A} \pm \frac{\mathbf{r}_{D}}{2} ; \xi_{A}\right) d^{2} \xi_{A},
$$

where $W\left(r_{A} \pm r_{D} / 2\right)=\mu\left(r_{A} \pm r_{D} / 2\right) \sqrt{S\left(r_{A}+r_{D} / 2\right)} \sqrt{S\left(r_{A}-r_{D} / 2\right)}$, with $\mu\left(r_{A} \pm r_{D} / 2\right)$ the complex degree of spatial coherence at the $O P, S\left(r_{A} \pm r_{D} / 2\right)$ the power spectrum recorded by the detectors (Mandel and Wolf, 1995). Interference terms between wavelets are not included in equation (3). It generates a moiré, which is called spatial coherence moiré (Castañeda, 2010a).

\section{Correlation between spatial coherence wavelets}

The following equation determines the correlation between the cross-spectral densities of the field at the $O P$ referred to the structured spatial coherence supports centered in the points $\mathrm{r}_{\mathrm{A}}$ and $\mathrm{r}_{A}^{\prime}$, respectively:

$$
G\left(\mathbf{r}_{A} \pm \frac{\mathbf{r}_{D}}{2}, \mathbf{r}_{A}^{\prime} \pm \frac{\mathbf{r}_{D}^{\prime}}{2}\right)=\left\langle W\left(\mathbf{r}_{A} \pm \frac{\mathbf{r}_{D}}{2}\right) W *\left(\mathbf{r}_{A}^{\prime} \pm \frac{\mathbf{r}_{D}^{\prime}}{2}\right)\right\rangle,
$$

with \langle\rangle denoting ensemble average. Thus, equations (3) and (4) load to the correlation between spatial coherence wavelets:

$$
\begin{aligned}
& G\left(\mathbf{r}_{A} \pm \frac{\mathbf{r}_{D}}{2}, \mathbf{r}_{A}^{\prime} \pm \frac{\mathbf{r}_{D}^{\prime}}{2}\right)=\left(\frac{1}{\lambda z}\right)^{4} \exp \left(i \frac{k}{z}\left(\mathbf{r}_{A} \cdot \mathbf{r}_{D}-\mathbf{r}_{A}^{\prime} \cdot \mathbf{r}_{D}^{\prime}\right)\right) \\
& \times \int_{A P A P}\left\langle\mathbf{W}\left(\mathbf{r}_{A} \pm \frac{\mathbf{r}_{D}}{2}, \xi_{A}\right) \mathbf{W} *\left(\mathbf{r}_{A}^{\prime} \pm \frac{\mathbf{r}_{D}^{\prime}}{2}, \xi_{A}^{\prime}\right)\right) d^{2} \xi_{A} d^{2} \xi_{A}^{\prime} .
\end{aligned}
$$

Thereby equations (1), (2) and (5) point out that the second-order spatial coherence state of the field at the $O P$, represented by $\left\langle W\left(r_{A} \pm r_{D} / 2\right) W^{*}\left(r_{A}^{\prime} \pm r_{D}^{\prime} / 2\right)\right\rangle$, results from contributions of the second-order spatial coherence state of the field at the $A P$, given by $\left\langle W\left(\xi_{A} \pm \xi_{D} / 2\right) W^{*}\left(\xi_{A}^{\prime} \pm \xi_{D}^{\prime} / 2\right)\right\rangle$, which are propagated by:

$$
\begin{aligned}
\mathbf{K}\left(\xi_{A}, \xi_{A}^{\prime}, \mathbf{r}_{A}, \mathbf{r}_{A}^{\prime}\right)=\left\langle\mathbf{S}\left(\xi_{A}, \mathbf{r}_{A}\right) \mathbf{S}\left(\xi_{A}^{\prime}, \mathbf{r}_{A}^{\prime}\right)\right\rangle \\
=\int_{A P} \int_{A P}\left\langle W\left(\xi_{A} \pm \frac{\xi_{D}}{2}\right) W^{*}\left(\xi_{A}^{\prime} \pm \frac{\xi_{D}^{\prime}}{2}\right)\right\rangle \\
\quad \times \exp \left[i \frac{k}{z}\left(\xi_{A} \cdot \xi_{D}-\xi_{A}^{\prime} \cdot \xi_{D}^{\prime}\right)\right] \\
\quad \times \exp \left[-i \frac{k}{Z}\left(\xi_{D} \cdot \mathbf{r}_{A}-\xi_{D}^{\prime} \cdot \mathbf{r}_{A}^{\prime}\right)\right] d^{2} \xi_{D} d^{2} \xi_{D}^{\prime},
\end{aligned}
$$


such propagation is depicted in Figure 2. Using equation (6), the new second-order spatial coherence wavelets that propagate the second-order spatial coherence state of the field can be expressed as:

$$
\begin{aligned}
& \mathbf{G}\left(\mathbf{r}_{A} \pm \frac{\mathbf{r}_{D}}{2}, \mathbf{r}_{A}^{\prime} \pm \frac{\mathbf{r}_{D}^{\prime}}{2} ; \xi_{A}, \xi_{A}^{\prime}\right)= \\
& \left\langle\mathbf{W}\left(\mathbf{r}_{A} \pm \frac{\mathbf{r}_{D}}{2}, \xi_{A}\right) \mathbf{W} *\left(\mathbf{r}_{A}^{\prime} \pm \frac{\mathbf{r}_{D}^{\prime}}{2}, \xi_{A}^{\prime}\right)\right)= \\
& \quad \mathbf{K}\left(\xi_{A}, \xi_{A}^{\prime}, \mathbf{r}_{A}, \mathbf{r}_{A}^{\prime}\right) \exp \left[-i \frac{k}{Z}\left(\xi_{A} \cdot \mathbf{r}_{D}-\xi_{A}^{\prime} \cdot \mathbf{r}_{D}^{\prime}\right)\right]
\end{aligned}
$$

With equation (7) in (5), the next expression is obtained:

$$
\begin{aligned}
& G\left(\mathbf{r}_{A} \pm \frac{\mathbf{r}_{D}}{2}, \mathbf{r}_{A}^{\prime} \pm \frac{\mathbf{r}_{D}^{\prime}}{2}\right)=\left(\frac{1}{\lambda z}\right)^{4} \exp \left[i \frac{k}{Z}\left(\mathbf{r}_{A} \cdot \mathbf{r}_{D}-\mathbf{r}_{A}^{\prime} \cdot \mathbf{r}_{D}^{\prime}\right)\right] \\
& \times \int_{A P} \int_{A P} \mathbf{K}\left(\xi_{A}, \xi_{A}^{\prime}, \mathbf{r}_{A}, \mathbf{r}_{A}^{\prime}\right) \exp \left[-i \frac{k}{Z}\left(\xi_{A} \cdot \mathbf{r}_{D}-\xi_{A}^{\prime} \cdot \mathbf{r}_{D}^{\prime}\right)\right] d^{2} \xi_{A} d^{2} \xi_{A}^{\prime} .
\end{aligned}
$$

The measurement of second-order correlation involves the combination of power spectrum values in a correlator. The power spectrum values are simultaneously recorded by two squared-modulus detectors, placed at two different points on the $O P$. The correlator is an electronic device that receives the signals from both detectors and multiplies them (Hanbury-Brown and Twiss R, 1956). A requirement of such measuring strategy is to arrange the detectors in the same structured support, i.e., $r_{A}=r_{A}^{\prime}$ and $r_{D}=r_{D}^{\prime}$, so that equation (8) becomes:

$$
\begin{aligned}
& G^{(2)}\left(\mathbf{r}_{A} \pm \frac{\mathbf{r}_{D}}{2}\right)=\left\langle\left|W\left(\mathbf{r}_{A} \pm \frac{\mathbf{r}_{D}}{2}\right)\right|^{2}\right\rangle=\left(\frac{1}{\lambda z}\right)^{4} \\
& \times \int_{A P} \int_{A P} \mathbf{K}\left(\xi_{A}, \xi_{A}^{\prime}, \mathbf{r}_{A}\right) \exp \left[-i \frac{k}{z}\left(\xi_{A}-\xi_{A}^{\prime}\right) \cdot \mathbf{r}_{D}\right] d^{2} \xi_{A} d^{2} \xi_{A}^{\prime} .
\end{aligned}
$$

\section{Second-order young's experiment}

The simplest configuration for analyzing second-order correlation of the field involves a pair of first-order virtual point sources at the second layer, each one turned on by a specific correlated pair of radiant point sources on the first layer. This configuration contains four co-linear radiant point sources on one-dimensional mask. Such situation is illustrated in Figure 3.

The four radiant point sources can emit different powers. The first and second source, and the third and fourth source have the same separation $a$, the second and third source have separation $b$. Therefore, the separation between the two first-order virtual point sources is $a+b$. These sources of the second layer turn on a second-order virtual point source at the third layer. It is assumed propagation in Fraunhofer domain and real-valued degree of first-order spatial coherence $\mu\left(\xi_{A} \pm \xi_{D} / 2\right)$.

The correlated pairs of radiant point sources are constituted by the first and the second source, and the third and fourth source, respectively. The coordinate origin of $A P$ is at the first source. Two squared-modulus detectors are placed at different positions on the $O P$, with separation $\mathrm{r}_{D}=x_{D}$ and coordinate origin $r_{A}=0$.

The first and second layers of $A P$ are involved with radiant energy and modulated energy, respectively. The third layer is associated with modulated coherence. The fourth layer represented in Figure 3 is the unified structure; this one contains the three types of point sources. The black bars denote the opaque segments of the mask and allow determining the relative positions of the different point sources. Note that the radiant point sources are always placed in the mask openings while the virtual point sources are at the midpoints of the opaque segments.

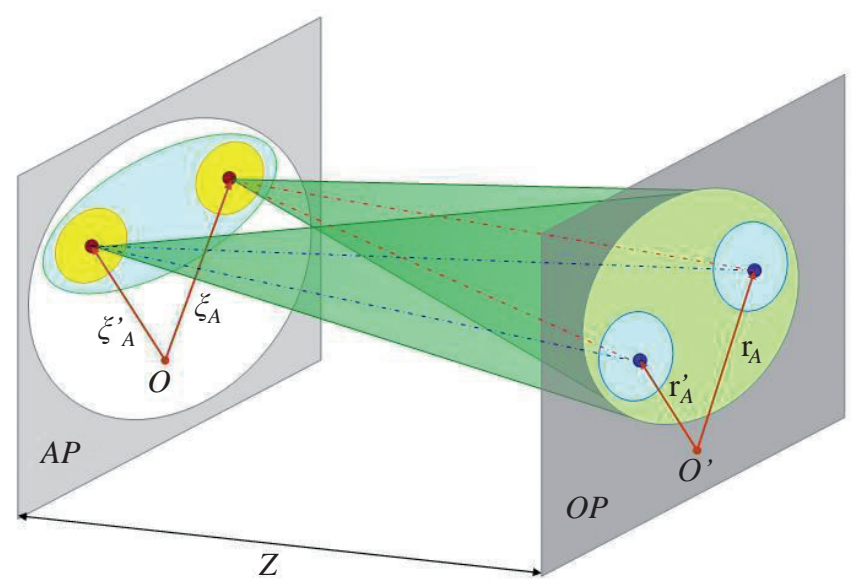

Figure 2. The cones represent the propagation of $\mathbf{G}\left(\mathrm{r}_{A} \pm \mathrm{r}_{D} / 2, \mathrm{r}_{A}^{\prime} \pm\right.$ $\left.\mathrm{r}_{D}^{\prime} / 2 ; \xi_{A}, \xi_{A}^{\prime}\right)$ from $A P$ to $O P$.

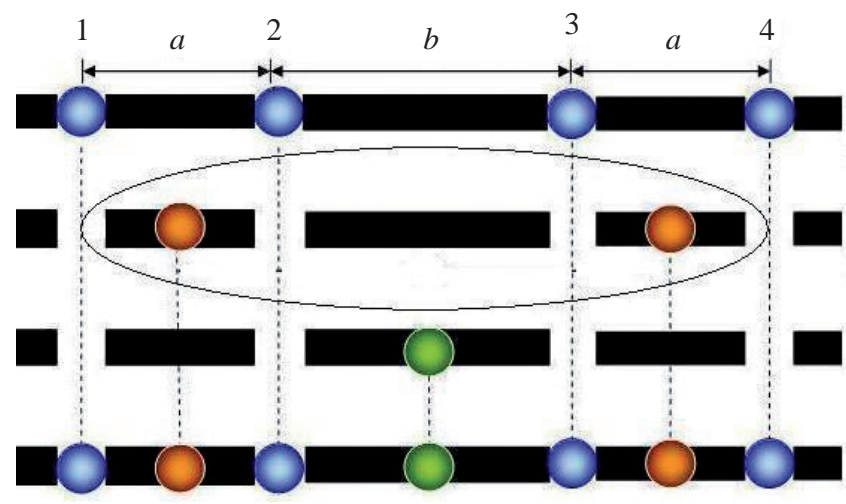

Figure 3. Diagram of layers at $A P$ for second-order Young's experiment. The black bars denote the opaque segments of the mask and the circles denote the point sources. There are four radiant point sources on first layer on top, placed at the mask openings, two first-order virtual point sources on second layer from top and a second-order virtual point source on third layer from top. The virtual point sources are at the midpoints of the opaque segments of the mask. The bottom layer is the unified structure. 


\section{Results}

The dimensionless functions $1 \equiv C \delta\left(\xi_{D}\right)+\left[1-C \delta\left(\xi_{D}\right)\right]$ and $1 \equiv C^{\prime} \delta\left(\xi_{D}^{\prime}\right)+\left[1-C^{\prime} \delta\left(\xi_{D}^{\prime}\right)\right]$ are introduced in order to separate the contributions of radiant and virtual point sources in the equation (9). It can be expressed like the sum of the terms:

$$
G_{\text {rad }}^{2}=\left(\frac{1}{\lambda z}\right)^{4}\left[\left\langle S^{2}(0)\right\rangle+\left\langle S^{2}(a)\right\rangle+\left\langle S^{2}(a+b)\right\rangle+\left\langle S^{2}(2 a+b)\right\rangle\right],
$$

and

$$
\begin{aligned}
G_{v i r}^{2}\left(x_{D}\right)= & \left(\frac{1}{\lambda z}\right)^{4}\left[\langle S(a) S(0)\rangle\left\langle\mu^{2}(a, 0)\right\rangle\right. \\
& \left.+\langle S(b+2 a) S(b+a)\rangle\left\langle\mu^{2}(b+2 a, b+a)\right\rangle\right] \\
& +\left(\frac{1}{\lambda z}\right)^{4} 2\langle\sqrt{S(a)} \sqrt{S(0)} \sqrt{S(b+2 a)} \sqrt{S(b+a)}\rangle \\
& \langle\mu(a, 0) \mu(b+2 a, b+a)\rangle \cos \left[\frac{k}{Z}(a+b) x_{D}\right] .
\end{aligned}
$$

$G_{\text {rad }}^{2}$ is the square of the power spectrum values recorded individually by the detectors; it is the contribution by the radiant point sources which does not depend on the $O P$ coordinates. The two first terms of $G_{\text {vir }}^{2}$ are the contributions due to the first-order virtual point sources while the last term is the contribution due to the second-order virtual source. This term results from the correlation between power spectrum values, so that the cosine modulation factor depends on the separations of both the second-order virtual point sources $a+b$ on $A P$, and the detectors $x_{D}$ on $O P$.

In order to relate these results with chaotic sources, for instance a binary star system, each correlated pair of radiant point sources can be associated to the ends of a star. Such analogy is often used in astronomy (Foellmi, 2009). Equation (11) depends on the separation $a$ of each pair of radiant point sources (angular separation $a / z$ of each star), the separation $a+b$ between pairs of radiant point sources (angular separation $(a+b) / z$ between a pair of stars) and separation $x_{D}$ of the detectors (telescopes).

The first two terms of equation (10) characterize the contribution of the two radiant point sources on the left in Figure 3 and the first term in equation (11) characterizes the virtual point source activated by them. These amounts represent the star on the left of the binary system. Similarly, the last two terms of equation (10) characterize the two radiant point sources on the right in Figure 3 and the second term of equation (11) their virtual point source. All these represent the star to the right of the binary system.

The virtual source of the third layer provides the cosine modulation on the measuring. This depends on the correlation between the two virtual sources of the second layer (correlation between stars). It is determined by the amount $\langle\mu(a, 0)+\mu(b+2 a, b+a)\rangle$. Then the above amount is zero in equation (11) for uncorrelated virtual point sources (i.e. uncorrelated system of independent stars).
Figure 4 shows the profiles of normalized $G_{v i r}^{2}$ by integrating equation (11) over all possible pairs of radiant point sources on the disk of each star (Hanbury-Brown, 1974), when the binary stars are: a) uncorrelated systems, b) correlated systems of identical elements (stars with same size and bright) and c) correlated systems with one element (star) three times brighter than the other. For the first instance, $G_{\text {vir }}^{2}$ is less than 1 in $x_{D}=0$, because the last term disappears in equation (11).
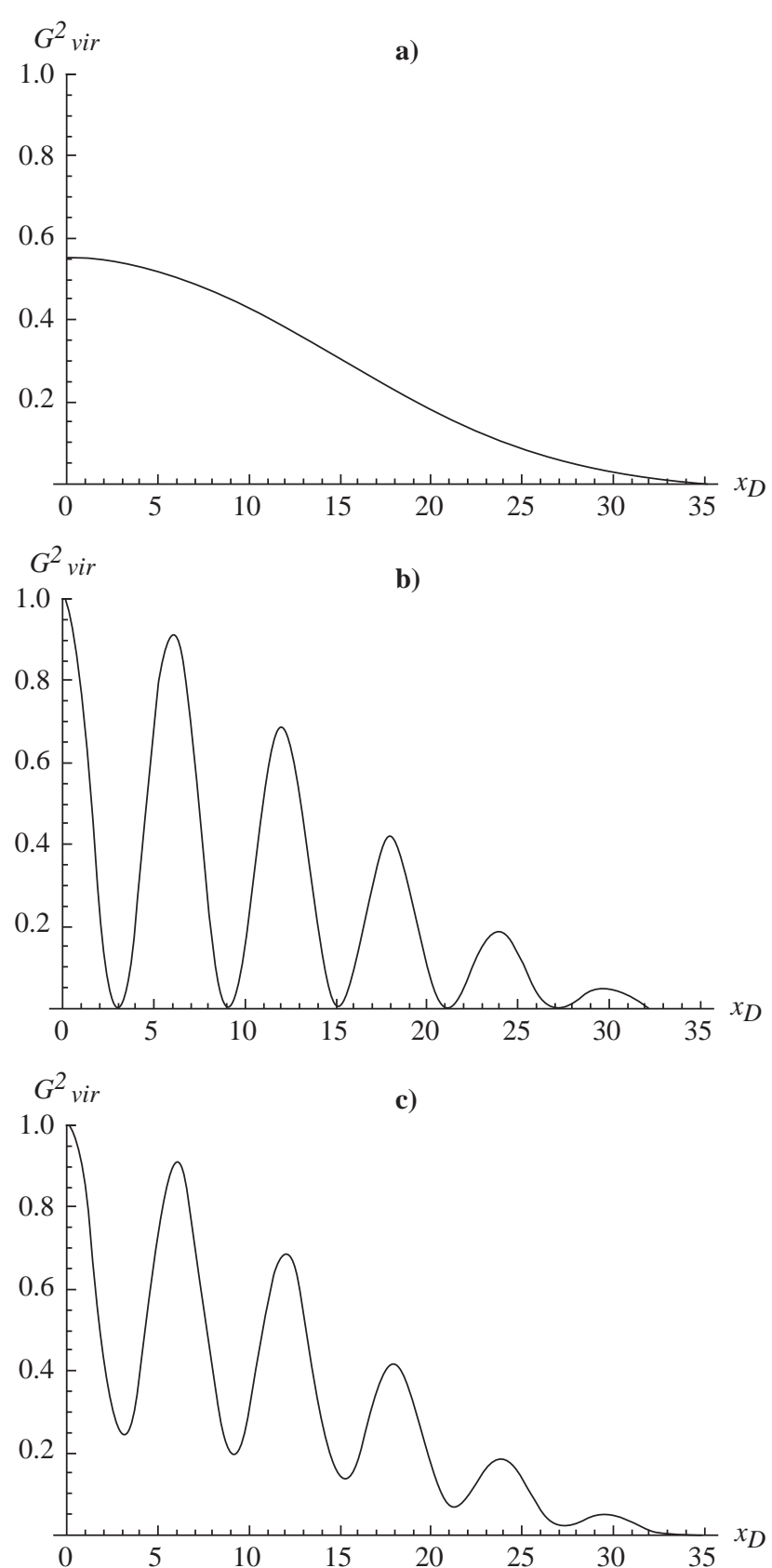

Figure 4. Schematics $G^{2}{ }_{\text {vir }}$ vs. $x_{D}$ obtained for second-order Young's experiments when the binary stars are: a) uncorrelated systems, b) correlated systems with identical elements and c) correlated systems with one element three times brighter than the other. 


\section{Hanbury-Brown and Twiss Effect}

The first measurement of the degree of second-order spatial coherence was performed by Hanbury-Brown and Twiss $(H B \& T)$ with the intensity interferometer. This type of interferometer measured the correlation of fluctuations of the intensities recorded at two points (telescopes) at the same time. They wanted to measure the angular separation of binary stars systems which could not be solved by other methods. HB\&T reported the following expression (Hanbury-Brown, et al., 1967):

$\Gamma^{2}(d)=\frac{1}{\left(I_{1}+I_{2}\right)^{2}}\left[I_{1}^{2} \Gamma_{1}^{2}+I_{2}^{2} \Gamma_{2}^{2}+2 I_{1} I_{2}\left|\Gamma_{1}\right|\left|\Gamma_{2}\right| \cos \left\{\frac{2 \pi \theta d}{\lambda} \cos \psi\right\}\right]$,

with $\Gamma^{2}$ as normalized degree of second-order spatial coherence, where $d$ is the distance between the telescopes (called baseline), $I_{1}$ and $I_{2}$ are intensities of the two stars,

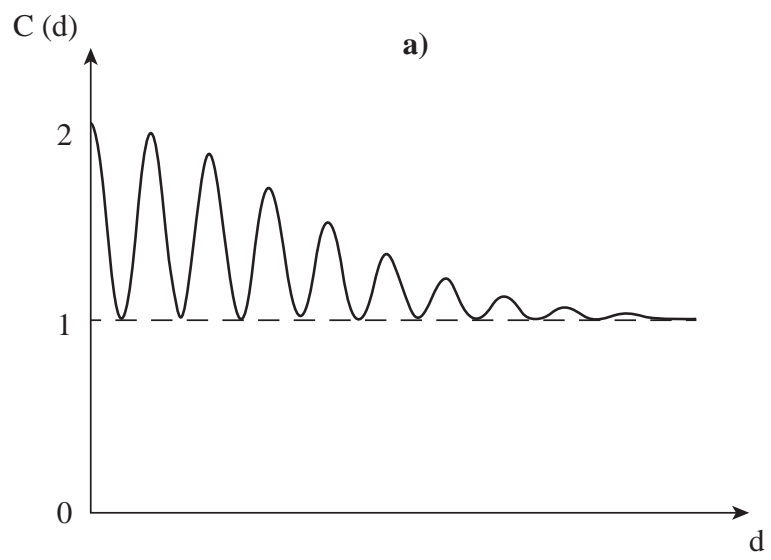

b)

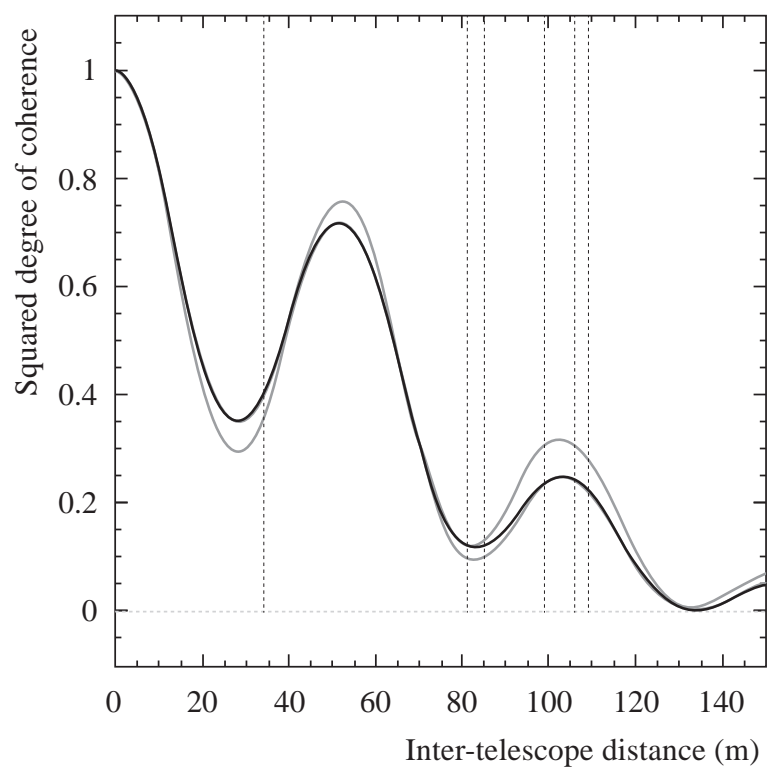

Figure 5. Modeled measures for degree of second-order spatial coherence in: a) binary system with identical stars, where $\mathrm{C}(\mathrm{d})=\Gamma^{2}$ (d) +1 (Baym, 1998) and b) binary stars Spica type when one star is brighter than the other (Le Bohec and Holder, 2006).
$\Gamma_{1}$ and $\Gamma_{2}$ are the degrees of first-order spatial coherence for each star, $\theta$ is the angular separation of the binary system, $\lambda$ is the chosen component of the light emitted by the stars and $\psi$ is the angle between the line joining of stars and the baseline. In this paper, $\psi$ is zero because of $A P$ and $O P$ are parallel. The equation (11) resembles the mathematical form of equation (12). The argument of the modulation is the same in both equations. The patterns given by equation (12) are plotted in Figure 5 when the binary stars are: a) correlated system of identical stars (Baym, 1998) and b) correlated system with one star brighter than the other (Le Bohec and Holder, 2006). The corresponding theoretical profiles in Figure 4 are in good (qualitative) agreement with the reported experimental curves in Figure 5. A quantitative fit of the theoretical profiles and the experimental curves has been not possible because the experimental data are reserved.

\section{Conclusion}

The second-order spatial coherence state of wave-fields can be analyzed and described in the framework of the classical wave picture and chaotic sources, through the second-order spatial coherence wavelets. This description leads to the correlation of power spectra on the observation plane, which is product of Young-like experiments with first-order virtual sources at the second layer. A secondorder virtual point source at a third layer is turned on because of the correlation between pairs of the firstorder virtual point sources. Such source is responsible of modulation on the coherence.

It suggests that the second-order spatial coherence state of light can be described in terms of three layers of point sources; a strategy that can increase the performance of numerical algorithms. Such modeling leads to a degree of second-order spatial coherence which is closely related to the result obtained by Hanbury-Brown and Twiss for binary stars.

\section{Acknowledgements}

I thank professor Román Castañeda (Universidad Nacional, Sede Medellín) for suggesting this interesting subject of work. I am grateful to Dr. Herbert Vinck Posada (Universidad Nacional, Sede Bogotá) for advices and suggestions. I wish to thank Dr. Gladys Villamarín (Universidad Autónoma, Bogotá) for economic supporting of activities of the international year of light.

\section{Conflict of interests}

The author has declared that no competing interests exist.

\section{References}

Baym, G. (1998). The physics of Hanbury Brown-Twiss intensity interferometry: from stars to nuclear collisions. Acta Physica Polonica B: 1839- 1884.

Castañeda, R. (2010). The Optics of the Spatial Coherence Wavelets. In Peter W. Hawkes, editor: Advances in Imaging and Electron Physics, 164: 29-255. 
Castañeda, R., et al. (2010). Radiant, virtual and dual sources of optical fields in any state of spatial coherence. J. Opt. Soc. Am. A, 27: 1322-1330.

Foellmi, C. (2009). Intensity interferometry and the secondorder correlation function in astrophysics. Astronomy \& Astrophysics, 11739-09.

Goodman, J. (2000). Statistical Optics. New York. Wiley Classics Library.

Hanbury-Brown, R. (1974). The intensity interferometer. New York, Halsted Press.
Hanbury-Brown R. and Twiss R. (1956). A test of a new type of stellar interferometer on Sirius. Nature, 178: 1046-1048

Hanbury-Brown R., Davis F., Allen L.R. and. Rome F.M. (1967). The Stellar Interferometer at Narrabri ObservatoryII. Mon. Not. R. Astr. Soc., 137: 393-417

Le Bohec, S. and Holder, J. (2006). Optical Intensity Interferometry with Atmospheric Cerenkov Telescope Arrays. The Astrophysical Journal, 649: 399-405

Mandel L. and Wolf E. (1995). Optical Coherence and Quantum Optics. Cambridge, Cambridge University Press.

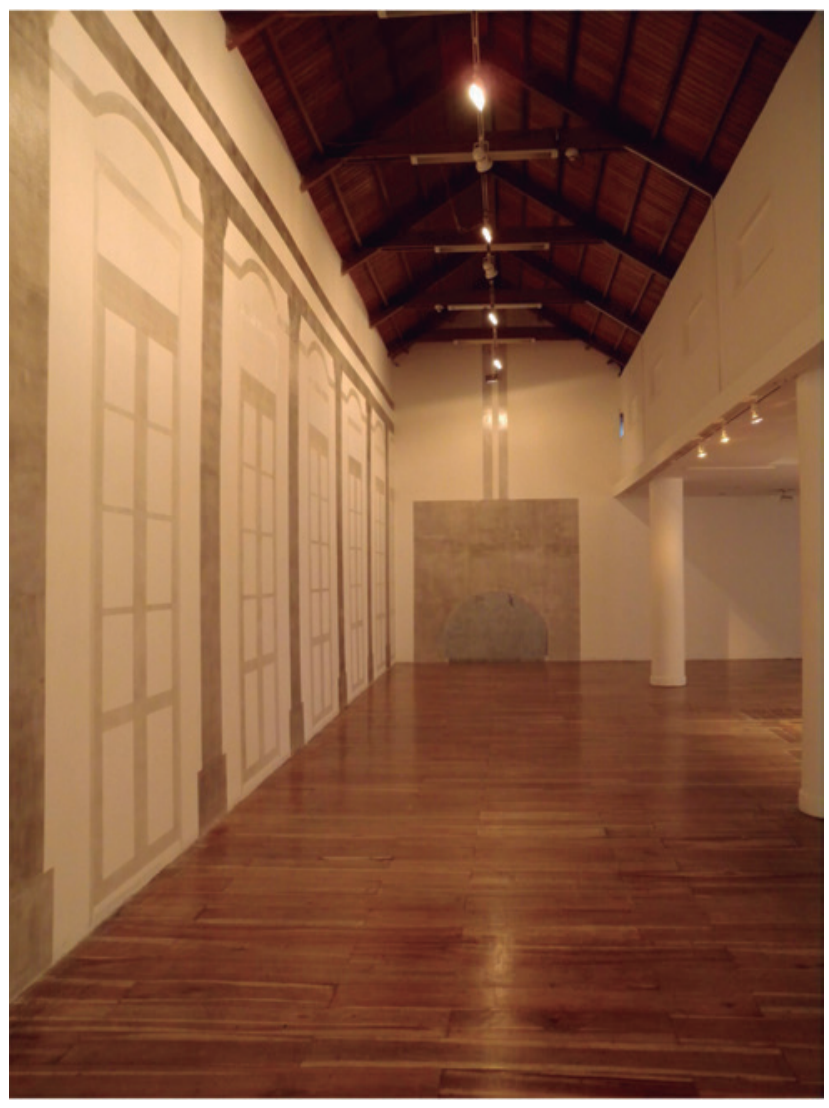

¿Es un imperio

esa luz que se apaga

o una luciérnaga?

Jorge Luis Borges (1899 - 1986) 